\title{
El espionaje y agencias de seguridad: los Estados Unidos y la Federación Rusa
}

\author{
Espionage and Security Agencies: The United States and the Russian Federation ${ }^{2}$
}

Daniela Alejandra Alba Useche ${ }^{3}$

CIENCIA Y PODER AÉREO

ISSN 1909-7050 | E-ISSN 2389-2468 |Volumen 9 | Enero-Diciembre de 2014 | Colombia | Pp. 97-105

Recibido: 13/08/2014

Aprobado evaluador interno: 15/09/2014

Aprobado evaluador externo: 09/11/2014

'Artículo de reflexión derivado del trabajo de grado titulado: Estados Unidos-Federación Rusa: espionaje y agencias de seguridad. Una mirada a las administraciones Bush-Putin Obama-Medvédev y Obama-Putin. Director: Prof. Douglas Eduardo Molina Orjuela.

2 Reflection article from the degree work titled: United StatesRussian Federation: Espionage and Security Agencies. An insight of Bush-Putin, Obama-Medvedev and Obama-Putin Administrations. Advisor: Prof. Douglas Eduardo Molina Orjuela.

${ }^{3}$ Internacionalista y Politóloga. Joven Investigadora del Grupo de Investigación Procesos de Integración, Regionalización y Estructuras Organizacionales (PIREO). Universidad Militar Nueva Granada. Correo electrónico: dannyalba_89@hotmail.com International Relations y Political Sciences graduate. Young Researcher of the Research Group for Integration, Regionalization and Organizational Structures (PIREO). Nueva Granada Military University.E-mail:dannyalba_89@hotmail.com
Resumen: La evolución del espionaje y la tecnología desde la Guerra Fría entre los Estados Unidos y la Federación Rusa se ha desarrollado a través de las Agencias de Seguridad, pero desde los ataques del 11-S, hubo un giro en los métodos de atacar el terrorismo; este tema se convirtió en la prioridad para Estados Unidos y para el resto del mundo. Por esta razón, se están desarrollando vertiginosamente programas de vigilancia, recopilación y análisis de información, sirviéndose del ciberespacio y del ciberespionaje, donde ahora todos somos observados y la privacidad ha sido reemplazada por la vigilancia 'en aras' de la seguridad.

Palabras clave: Agencias de seguridad, espionaje, Estados Unidos, Federación Rusa, información, seguridad nacional.

Abstract: The evolution of espionage and technology since the Cold War between the United States and the Russian Federation has been developed through the security agencies, but since September 11 attacks, there was a shift in the methods of combating terrorism; this issue became a priority for the United States and the rest of the world. For this reason there are rapidly developing programs for monitoring, gathering and analyzing of information, making use of cyberspace and cyber espionage, where we are all observed and privacy has been replaced by surveillance for safety's 'sake'.

Key Words: Espionage, Information, National Security, Russian Federation, Security Agencies, United States. 


\section{Introducción}

En el contexto de las Relaciones Internacionales, las potencias han permanecido en conflicto por intereses económicos, geopolíticos, militares y tecnológicos, desde las prácticas tradicionales de espionaje utilizadas en la Guerra Fría, y que en la actualidad son desarrolladas a través de las agencias de seguridad e inteligencia, con grandes avances en tecnología; aportando mayor eficacia en el control de la información, donde el ciberespionaje se convierte en un arma poderosa, silenciosa y efectiva.

Así el ciberespionaje ha tomado fuerza y es un asunto de suma importancia para ser tratado por los gobiernos y actores internacionales como los Estados Unidos, que tiene en su poder las tecnologías más avanzadas de vigilancia y control de la información de casi todo aquel que tenga un computador o un celular.

En la apreciación de la Federación Rusa, según Morelli (2013):

Para Estados Unidos las tecnologías de la información son una herramienta importante para potenciar su poderío militar y les gustaría permanecer libres del control para poder usarlas a discreción en aplicaciones militares y políticas y quedar fuera de las regulaciones del Derecho Internacional (p.7).

En la política internacional, una de las prioridades es mantener buenas relaciones con los demás actores del Sistema Internacional. Sin embargo, se han visto vulneradas por el ciberespionaje, creando un sinnúmero de agencias de seguridad especializadas en control terrestre, aéreo, marítimo, informático y espacial. Inicialmente, la única forma de obtener información era infiltrando agentes en instituciones gubernamentales; ahora el espionaje se sirve de la tecnología, donde el nuevo método de recopilación de información es el ciberespionaje y la tecnología se ha convertido en un arma muy poderosa.

Pero el principal problema que representa esta herramienta, es que todos los usuarios de este tipo de tecnologías se ven afectados por los programas encaminados a mejorar la Seguridad Nacional, cuyo objetivo es proteger a los ciudadanos del territorio nacional de cualquier amenaza, sea interna o externa. Un ejemplo de estas estrategias de Seguridad Nacional es la propuesta por los Estados Unidos, que en el año 2002 fue modificada por el entonces presidente, George W. Bush, luego de los ataques del 11-S, recurriendo a las Fuerzas Armadas, diplomacia internacional y a la tecnología por medio de la Ley Patriota, creada para tener acceso a la información de todo lo que represente un delito de terrorismo con el objetivo de ingresar a Afganistán para buscar a su entonces enemigo, Osama Bin Laden y Al-Qaeda.

Debido al auge de estas nuevas tecnologías, los principales servidores de la Internet como Google, Facebook, Apple, Yahoo, entre otros, se unieron para realizar métodos de vigilancia con el fin de proteger y preservar la seguridad de los ciudadanos estadounidenses, a la vez que avanza un nuevo orden internacional.

El objetivo del presente artículo es entender los nuevos sistemas de guerra de los Estados Unidos y la Federación Rusa que se dan a partir del rol de las agencias de seguridad, mediante el uso de espionaje con ayuda de las nuevas tecnologías desde la Guerra Fría, además de hacer una mirada a las administraciones Bush-Putin, Obama-Medvédev y Obama-Putin.

Paralelo a este escenario, se observa el papel de las agencias de seguridad, donde la estrategia central es la inteligencia, y cómo se acuerdan las alianzas en aras de la seguridad; en el marco de tecnologías de control de la información, a través del ciberespionaje, en el que la privacidad pasa a un segundo plano, por la prioridad de la Seguridad Nacional.

\section{Método}

La metodología utilizada en el trabajo de investigación y artículo es cualitativa, pues su contenido produce datos descriptivos. En cuanto a la técnica de recolección, se hizo revisión de numerosos documentos con el objeto de recopilar información que conduzca a entender, aclarar y apoyar el tema sobre cómo a través de las confrontaciones entre Estados; los escenarios y los intereses de poder cambian, apelando al espionaje, las agencias de seguridad e inteligencia, las alianzas estratégicas y el desarrollo de tecnologías.

Para el análisis documental, se seleccionó y clasificó la información que fuera verificable, tuviera credibilidad y se aplicara al tema que incluye antecedentes, y descripciones de gran importancia. 


\section{Espionaje en la Guerra Fría}

Durante la Guerra Fría, las dos potencias del momento (Estados Unidos y URSS), vivieron un período en el cual la tecnología avanzaba de tal manera que se convertía en el nuevo elemento indispensable para realizar operaciones de espionaje; una herramienta de gran importancia para los Estados Unidos y la Unión Soviética.

En los inicios de la Guerra Fría en 1947, la Ley de Seguridad Nacional de los Estados Unidos creó el Director Central de Inteligencia (DCl). Este lideraba los servicios de inteligencia de los Estados Unidos y proporcionaba información a los funcionarios federales. La Agencia Central de Inteligencia (CIA, por sus siglas en inglés), como organismo independiente se encargaba de almacenar, analizar y entregar información al presidente, a través del Director de Inteligencia Nacional.

"La CIA fue creada originalmente para recoger información para realizar las estimaciones de inteligencia destinadas a los decidores políticos pero, sin pretender exculpar a la agencia de sus responsabilidades." (Barrientos Ramírez, 2010, p. 73). Como dice Carmen Medina, "en la Dirección de Inteligencia de la CIA, el análisis político sigue siendo todavía el rey" (Como se cita en Barrientos Ramírez, 2010, p. 77), ejecutado este análisis por el Comité Especial de Inteligencia del Senado y el Comité Permanente de Inteligencia de la Cámara de Representantes.

La Guerra Fría llega a su fin y los Estados Unidos toma medidas drásticas, debido a que no previeron la caída de la URSS. Con este argumento, iniciaron cambios radicales en sus estructuras de inteligencia y evitar así posibles nuevas amenazas.

A su vez, como consecuencia de la desintegración de la URSS, se creó Echelon; un programa de espionaje entre los Estados Unidos y Gran Bretaña; el cuál "tiene su origen en el acuerdo semi-secreto UK-USA (Gran Bretaña-Estados Unidos) firmado inicialmente en 1947, teniendo como principal cliente la CIA" (Pachón Ovalle, 2004, p. 2); la red secreta Echelon se creó a principios de los años 70 y fue ampliada y fortalecida entre los años 1975 y 1995. "Está dirigida por la NSA (National Security Agency) norteamericana y la Agencia Británica de Comunicaciones Gubernamentales (GCHQ)" (Pachón Ovalle, 2004, p. 2).

Este programa fue creado "para compartir inteligencia de señales entre las dos naciones y los socios de la
Commonwealth de Gran Bretaña, Canadá, Australia y Nueva Zelanda" (Burghardt, 2013). Echelon no sólo está destinado para recolectar e interceptar información, sino que también "se ha apelado a procedimientos informatizados de reconocimiento de voz y de texto, y de búsqueda de palabras." (Pachón Ovalle, 2004, p 2).

No sólo se encarga de interceptar comunicaciones, también de aplicar operaciones de reconocimiento de voz, ya que "en inteligencia artificial se filtran hasta 3.000 millones de mensajes por hora. Los diccionarios son manejados por la NSA" (Maldonado, 2013, p. 30). Llamados diccionarios porque se almacenan palabras clave. Ejemplo: guerra, presidente, bomba, ataque, entre otras.

"Como red es un entramado de antenas, estaciones de escucha, radares, satélites, submarinos y aviones espía, unidos todos estos elementos a través de bases terrestres, cuyo objetivo es espiar las comunicaciones mundiales" (Pachón Ovalle, 2004, p. 3). Otro método utilizado es el reconocimiento facial empleado a través de las cámaras de seguridad; todas estas tecnologías siguen siendo apoyadas por el espionaje humano; "los espías que se denominan 'durmientes' (sleepers), y que durante un tiempo largo hacen un trabajo soterrado de espionaje y doble inteligencia" (Maldonado, 2013, p. 31), infiltrando personas en empresas en ciertas labores, para hacer seguimientos y obtener la información deseada.

Echelon, le ha dado ventajas a los Estados Unidos en cuanto a espionaje,"su objetivo inicial era controlar las comunicaciones militares y diplomáticas de la Unión Soviética y sus aliados" (Sánchez Medero, 2009, p. 27); Echelon, era el programa más conocido en ese momento.

La agencia de seguridad que controla a Echelon es la NSA,"con sede en Fort Meade (Maryland), perteneciente al Departamento de Defensa" (Duque Quicios, 2006, p. 27), creada en 1957 y reconocida oficialmente en 1977, adscrita al Departamento de Defensa, su poderío está en que produce la mayoría de la inteligencia operativa dentro de la comunidad de inteligencia. Compuesta por profesionales calificados en ingeniería, matemática, programación, entre otros. "Se encarga de la protección de todos los servicios de información de la nación, así como de la recogida y análisis de todo tipo de señales que se lancen al espacio" (Duque Quicios, 2006, p. 27). Toda comunicación extranjera es interceptada, analizada y decodificada por ésta agencia. 
De ésta manera, la Guerra Fría fue una oportunidad para poner a prueba la nueva tecnología en las comunicaciones, por lo que se aprovechó el uso de Echelon, donde el objetivo ya no es buscar un bien material o un arma peligrosa, sino que se busca algo más importante e impalpable; la información, que ha sido parte esencial en todos los escenarios del mundo, por lo que la infoesfera (el mundo comunicado) Ilamada así por el sociólogo Alvin Toffler desde la Guerra Fría, que aparentemente ya terminó. El objetivo de los Estados y de las agencias de espionaje en el mundo es ahora la información como arma de poder.

\section{Espionaje en la Post- Guerra Fría}

El fin de la Guerra Fría, acontecida entre 1989 y 1991, dejó un escenario de confusión en el mundo; la Unión de Repúblicas Socialistas Soviéticas (URSS) se desintegra y paralelamente se hace un intento de acabar con el monopolio de la seguridad soviética por medio de la creación de nuevos organismos y agencias de seguridad desligadas al KGВ (КОМИT́ЕТ ГОСУД́АРСТВЕННОЙ БE3Oగ́AСНОСТИ, en español Comité para la Seguridad del Estado), pero conservando la misma estructura y que serviría como objetivo para preservar el poder.

También, prevalece el deseo de investigar qué tipo de métodos de inteligencia y seguridad poseen las naciones poderosas como los Estados Unidos. Vladimir Putin, en el pasado como agente del KGB, se mantiene el interés acerca de este tema. Se vale de la experiencia de los servicios de seguridad, conformados en su mayoría por los sucesores del KGB en cargos para labores de inteligencia, contrainteligencia, protección de fronteras, arsenal nuclear, entre otras.

Durante la Guerra Fría, las operaciones de espionaje entre el KGB y la CIA, aparentemente eran cosas del pasado, pero realmente lo que se impondría en el mundo sería el espionaje a través del ciberespacio que se empezaba a desarrollar después de la Segunda Guerra Mundial. Se hace realidad lo que en 1984 advertía George Orwell con el Gran Hermano, que todo lo ve, todo lo vigila, y todo lo controla. Estados Unidos, autoproclamado como el país de la libertad, es ahora uno de los pioneros en la creación de programas de vigilancia que en un inicio estaban pensados en proteger la Seguridad Nacional, pero que vulneran la privacidad individual, pues han incluido en sus objetivos a toda la población civil.
Por tal razón, la Ex-Unión Soviética creó una ley que le permite al SVR (Servicio de Inteligencia Exterior por sus siglas en inglés), "recabar información de carácter económico, científico y técnico; su principal destinatario podría ser para la industria de Defensa, que se encontraba en una profunda crisis tras el fin de la Guerra Fría" (Morales Hernández, 2003, p. 12), así como los temas de narcotráfico, Armas de Destrucción Masiva (MDW, por sus siglas en inglés) y terrorismo internacional, además de la prohibición de espionaje a ciudadanos rusos.

Comenzaba la era del unilateralismo al mando de Estados Unidos y sus agencias de seguridad que poco a poco conquistarían no sólo el territorio americano sino todo el globo. Echelon desde la Guerra Fría se convirtió en "una acción político-militar de gran envergadura, dictada por USA, sobre los sistemas de comunicación electrónica en el planeta, inicialmente justificada con el argumento de la Seguridad Nacional" (Pachón Ovalle, 2004, p. 1), pero que trasciende más allá de las fronteras.

De otra parte, en cuanto a la lucha contra el terrorismo internacional, la Federación Rusa tiene prevención con sus secretos de Estado; pero el electo presidente a principios de 2000, Vladimir Putin, había decidido "la posibilidad de cooperar con los servicios extranjeros para operaciones antiterroristas" (Morales Hernández, 2003, p. 5). Al iniciar su gobierno se aprueba un documento de la Doctrina de Seguridad Nacional, en la que alude a la seguridad de la información y el uso de los servicios de seguridad e inteligencia para resistir los ataques, que se desarrollarían en los Estados Unidos sobre guerra de información.

\section{Espionaje Post 11-S}

Los ataques del 11 de septiembre de 2001 cambiaron la agenda de Seguridad Nacional de los Estados Unidos y exponencialmente cambió el modo de combatir al enemigo. El Congreso aprueba el 'Patriot Act' o Ley Patriota, exactamente un mes después de los ataques terroristas que sacudieron a los Estados Unidos. Con esta nueva ley se les permite a los empleados de las agencias federales de seguridad, el poder de interceptar y recopilar comunicaciones para combatir la guerra contra el terrorismo, denominada así por el Presidente Bush el 14 de septiembre del mismo año. Posteriormente, en una declaración del 20 de septiembre de 2001, ofreció un discurso donde da a conocer el plan para desarrollar la guerra contra el terrorismo: 
Privaremos a los terroristas de financiamiento, pondremos a los unos contra los otros, los haremos ir de un lugar a otro, hasta que no haya refugio o descanso. $Y$ perseguiremos a las naciones que ayuden o den refugio al terrorismo. Toda nación en toda región del mundo, ahora tiene que tomar una decisión. Están de nuestro lado, o están del lado de los terroristas. A partir de hoy, cualquier nación que continúe albergando o apoyando al terrorismo será considerada un régimen hostil por los Estados Unidos. (Bush, Discurso ante una Sesión Conjunta del Congreso y El Pueblo Estadounidense, 2001).

Estados Unidos tenía planeado utilizar soldados para buscar a su enemigo que en ese entonces era Osama Bin Laden y el grupo terrorista Al-Qaeda, pero poseían una herramienta que podría ayudarlos de manera eficiente. La CIA, NSA y DIA (Agencia de Inteligencia en Defensa), aunque fueron creadas para evitar este tipo de ataques, no cumplieron la función para la que fueron creadas, ya que no pudieron evitar el ataque al World Trade Center y al Pentágono, y anticiparse a los movimientos de su nuevo enemigo.

A partir de ese suceso, el programa Echelon se convirtió en el mayor sistema de espionaje de los Estados Unidos, bajo el control de la NSA, que aunque no es el único programa de espionaje, es el que más operaciones ha hecho en el mundo. La creación de este programa inició en 1946, durante la Guerra Fría, mientras los Estados Unidos forma un acuerdo con Gran Bretaña "para compartir inteligencia de señales entre las dos naciones y los socios de la Commonwealth de Gran Bretaña, Canadá, Australia y Nueva Zelanda, conocido como el acuerdo de 'Cinco Ojos' " (Burghardt, 2013, p. 34).

Tras los hechos del 11-S, el mundo ha orientado su atención a la seguridad e inteligencia, incrementando recursos económicos para su desarrollo y la Federación Rusa no fue la excepción; El presidente Vladimir Putin mantenía dentro de la Unión Soviética una red de informadores para neutralizar actividades extranjeras e internas contra el régimen soviético, los cuales por su experiencia sirven a la Federación Rusa en los órganos de seguridad e inteligencia.

La obsesión por la Seguridad Nacional después del 11-S, tuvo que ser aplacada por Putin hacia sus ciudadanos piara abandonar la idea de ser víctimas de espionaje por parte del gobierno, debido a la gran cantidad de agencias secretas de inteligencia, al servicio de su presidente.

Son muchas las agencias de servicios de seguridad nacionales con que cuenta la Federación Rusa para preservar la soberanía y el poder, entre las que se encuentran: el GRU (Главное разведывательное управление, en español Departamento Central de Inteligencia), depende del Estado mayor y está encargado de la inteligencia militar y el Ministerio del Interior (MVD por sus siglas en inglés), posee unidades antiterroristas y tropas propias.

Otros organismos que dependen del presidente ruso y tienen algún origen en el KGB son: Consejo de Seguridad de la Federación Rusa, entidad que lo asesora en materia de políticas de seguridad; el Servicio Federal de Seguridad (FSB); el Ministerio del Interior, encargado de la seguridad interior; el Servicio Federal de Guardias de Fronteras (FPS por sus siglas en inglés) encargado de la vigilancia de las fronteras; el Servicio de Seguridad Presidencial (SBP por sus siglas en inglés), encargado de la seguridad del presidente; el SVR y la Agencia Federal de Comunicaciones Gubernamentales e Información (FAPSI por sus siglas en inglés), encargado de la criptografía, entre otras.

La FAPSI, se ocupaba de la inteligencia de señales, imágenes, electrónica, comunicaciones y criptografía, equivalente a la NSA, se cree que estaba compuesta entre 53.000 y 120.000 personas; "prohibía revelar cualquier dato acerca de sus capacidades y operaciones" (Morales Hernández, 2003, p. 14). Así mismo, controlaba toda información estatal, como lo afirmaba Morales Hernández (2003) "la red que permite la transmisión de los resultados electorales a Moscú, o la Intranet gubernamental llamada RGIN" (p. 14).

El presidente Putin decretó la terminación de la FAPSI y el FPS, fusionándolos a la agencia FSB y a su vez, el MVD se ocupó de la seguridad interior y persecución de delitos fiscales.

\section{Relaciones Estados Unidos- Federación Rusa Gobierno George W. Bush - Vladimir Putin}

A pesar que las relaciones entre la Federación Rusa y Estados Unidos han sido tensas, luego de los hechos ocurridos el 11 de septiembre de 2001, se unieron para realizar acuerdos de seguridad; la Federación Rusa apoyó la entrada de los Estados Unidos a Afganistán, per- 
mitiendo el ingreso de tropas a su territorio y al espacio aéreo Ruso; también autorizó la coordinación con las repúblicas centroasiáticas (Kazajistán, Kirguistán, Tayikistán, Turkmenistán y Uzbekistán) para éste apoyo a Estados Unidos, así como la participación en misiones de búsqueda y rescate y cooperación al gobierno afgano de Burhanuddin Rabban.

El apoyo de la Federación Rusa a los Estados Unidos con la operación "libertad duradera" en el ámbito diplomático, se vio positivamente en la actitud de China e Irán, en el Foro de Cooperación Económica Asia-Pacífico (APEC) o la Organización de Cooperación de Shangai (OCS).

La cooperación tuvo un resultado exitoso, tanto que "la sintonía entre Moscú y Washington se mantuvo varios años, permitiendo el establecimiento de bases estadounidenses y de otros países de la OTAN (Organización del Tratado del Atlántico Norte) no sólo en Asia, sino incluso en la díscola Georgia" (Serra, 2008, p. 87). Además, la situación de tensión en Oriente Medio ayudó a la Federación Rusa a mejorar su economía, triplicando el precio del barril de petróleo en 2003.

Los Estados Unidos veía con buenos ojos un acercamiento a la Federación Rusa, pues a nivel regional este país sobresale por su extensión territorial y sus riquezas energéticas, además de su poder de veto en el Consejo de Seguridad de las Naciones Unidas. El momento de tensión que vivieron estos dos países durante la Guerra Fría estaba desapareciendo; ahora pasaban por un buen momento, manteniendo un equilibrio de poder en el Sistema Internacional a pesar de la unipolaridad estadounidense, que se ha mantenido siempre, y como potencia quiere ser el actor decisivo en los conflictos.

A final del gobierno de G.W Bush empieza a funcionar un programa de vigilancia electrónica complementando a Echelon y creado por la NSA Ilamado PRISM, "que permite a los funcionarios recoger el material que incluye el historial de búsqueda, el contenido de mensajes de correo electrónico, transferencia de archivos y chats en vivo" (Greenwald \& McAskill, 2013), todo tipo de comunicaciones que las personas realicen pueden ser vigiladas por este programa que entró en vigencia desde 2007, y que siendo aprobado por el Congreso estadounidense le permite realizar éstas operaciones de vigilancia. Las acciones realizadas por PRISM son legales por medio de la ley de control de inteligencia extranjera (FISA por sus siglas en inglés) de
1978 aprobada por el Congreso, que permite la autorización de realizar escuchas para defender la Seguridad Nacional.

El programa ha causado controversia porque ha salido a la luz que está capacitado para acceder a la información de todos los usuarios de reconocidos servidores como "Microsoft, Yahoo, Google, Facebook, PalTalk, AOL, Skype, YouTube y Apple." (Ramonet, 2013, p. 2), que son direccionados a la NSA con el fin archivar, clasificar, analizar y usar para Inteligencia con propósito de salvaguardar la Seguridad Nacional.

El programa Echelon en la actualidad funciona con base en PRISM, que es un programa reciente y secreto de la NSA, sucesor de Carnivore de Microsoft en 1997, y que en el 2005 se reemplazó por NarusInsight. Paralelo a estos programas, han surgido múltiples agencias especializadas en todos los escenarios para prevención del terrorismo o cualquier ataque, sea por tierra, mar o aire; entre otras están:

La Agencia para la Reforma de la Inteligencia y la Prevención del Terrorismo (IRPA, por sus siglas en inglés), de los Estados Unidos, creada en 2004.

Homeland Security Department o Seguridad Nacional (HSD por sus siglas en inglés), está compuesta por más de 22 agencias de inteligencia y los centros antiterroristas. Se creó a partir del 11-S y cuenta con más de 185.000 empleados que dependen del jefe de Estado.

La Oficina Nacional de Reconocimiento, National Reconnaissance Office (NRO), dentro del Departamento de Defensa, coordina la obtención y análisis de la información de todos los satélites y aeronaves para todos los servicios de inteligencia y militares (Duque Quicios, 2006, p. 28).

La NGA (Agencia Nacional de Inteligencia Geoespacial por sus siglas en inglés), provee mapas de navegación terrestre, aérea, y todo tipo de herramientas de vigilancia. También se encarga de la inteligencia geoespacial, recopilando y analizando imágenes.

La CIO (Oficina Central de Imágenes, por sus siglas en inglés), forma parte del Departamento de Defensa y se encarga de la cartografía para el mismo departamento, la CIA y mandos de combate, entre otras. 
A su vez, el espionaje que se ha desarrollado durante décadas sigue vigente, es decir el humano (Humint), a través de agentes encubiertos, como periodistas, diplomáticos, activistas, entre otros, que recaban información y se exponen a ser detenidos. Pero el tipo de espionaje que no permite dejar huella, ni se conoce quién es el enemigo, ni la intención que este tiene, sigue creciendo; el ciberespionaje, un arma donde cualquiera puede ser un objetivo y es más difícil de detectar.

El FSB de la Federación Rusa, ha perseguido a supuestos colaboradores del espionaje, entre ellos muchos diplomáticos, ciudadanos extranjeros y los miembros de peace corps, una organización humanitaria del gobierno estadounidense, acusados de espionaje. Los métodos seguirán cambiando, pero el fin es el mismo, neutralizar las acciones del enemigo.

\section{Gobierno Barack Obama - Dmitri Medvédev}

La relación durante la Administración Medvédev con Obama inició con un poco de distanciamiento y frialdad; Obama por su parte llegó a Europa y Asia con ansias de cambiar la relación que se había construido con su predecesor G.W. Bush. Al presidente norteamericano le interesaba retomar las relaciones con la Federación Rusa, porque sabe que la imagen en la región es fuerte y"le ha permitido parar en seco los proyectos de expansión de la democracia en la zona y desafiar la política estadounidense en otras regiones del globo" (Tovar Ruiz, 2009, p. 5).

Los Estados Unidos y la Federación Rusa atravesaron algunos problemas como la situación con "las fronteras de Georgia, y la hipotética incorporación a la OTAN (Organización del Tratado del Atlántico Norte), forzada por los Estados Unidos, de este país y de Ucrania (cuya población rechaza el ingreso)" (Polo, 2009, p. 43). Además de las disputas que se vinculan con la explotación de hidrocarburos en la zona del Caspio y Asia central, que persisten hasta la fecha de la publicación del presente texto.

Dejando a un lado las diferencias entre Obama y Medvédev, este último desea realizar cambios a nivel económico en la Federación Rusa y por medio de los Estados Unidos "espera recibir las inversiones y tecnología necesarias para modernizar la economía rusa" (de la Cámara, 2010, p. 2). Por su parte, el Presidente Obama se siente cómodo con la llegada de Medvédev, logrando un "reset o reconfiguración de las relaciones entre Washington y Moscú, que abre perspectivas prometedoras" (De la Cámara, 2010, p. 2).
La relación entre Moscú y Washington durante este período mejoró notablemente, teniendo en cuenta las diferencias del anterior gobierno con George W. Bush y Vladimir Putin como los actores principales. Hubo una tendencia a la cooperación de forma recíproca; los Estados Unidos busca de la Federación Rusa apoyo "en los grandes temas de la agenda internacional con Irán, Afganistán o la proliferación nuclear" (De la Cámara, 2010, p. 21).

Aprovechando el buen momento con la Administración Obama, el Presidente Medvédev realizó un viaje a "Silicon Valley para estudiar el modelo de desarrollo de industrias de alta tecnología" (de la Cámara, 2010, p. 22). Asimismo, mostrar su interés por el modelo que utiliza el gobierno de los Estados Unidos y la Agencia de Proyectos de Investigación Avanzados de Defensa (DARPA por sus siglas en inglés) a nivel tecnológico y de innovación conjunta con el Ejército y la policía.

\section{Barack Obama - Vladimir Putin}

La relación entre estos dos jefes de Estado no tuvo un buen comienzo a nivel político; las elecciones en el año 2011 en la Federación Rusa para elegir al Presidente, se vieron nubladas por opiniones del Partido Comunista y medios de comunicación Europeos y Norteamericanos, quienes aseguran que hubo fraude electoral y represión por parte de la policía a los manifestantes que mostraron desacuerdos en los resultados electorales.

Por su parte, Vladimir Putin denunció a los Estados Unidos de buscar "la desestabilización de Rusia, criticó las declaraciones públicas de Hillary Clinton, y aseguró que "centenares de millones" de fondos extranjeros están estimulando protestas y financiando organizaciones" (Polo, 2012, p. 28), encaminadas a falsear la imagen de Putin hacia occidente, por fraude electoral, pero es claro que las dos naciones no sólo tienen choques en lo político, también lo es a nivel diplomático y de seguridad.

Más adelante, en los Estados Unidos durante el gobierno de Barack Obama, estalla el escándalo de ciberespionaje de grandes proporciones que ha conmocionado a gobernantes y a la población, acerca de alianzas y acuerdos entre el gobierno estadounidense y grandes corporaciones para vigilar y obtener información proveniente de las grandes empresas proveedoras de Internet y de todos los usuarios.

Este caso ha amenazado la Seguridad Nacional y global; y dada la importancia de mantener la información 
confidencial segura de potenciales enemigos; muchas personas trabajan en pro de salvaguardar estos intereses. Sin embargo, no siempre existe lealtad de los empleados con el gobierno y publican información que podría ser el talón de Aquiles para el país. Edward Snowden, ex-agente de la CIA, quien durante mucho tiempo reunió evidencia de información secreta, decide filtrar información convirtiéndose en informante o whistleblower, para revelar al mundo el secreto acerca de las interceptaciones que el gobierno de los Estados Unidos por medio de la NSA y otras agencias de inteligencia realizan sin consentimiento a las naciones y a primeros ministros de Europa, América Latina y casi el mundo entero.

Entre los documentos revelados por Snowden, se conoce que una de las prioridades de Washington dentro de las agencias de seguridad es hablar otros idiomas, principalmente el ruso, lo cual es retribuido económicamente, en este caso, a 736 espías por los servicios de inteligencia estadounidenses. "El aparato de espionaje de la Casa Blanca incluye como objetivos prioritarios a Rusia, China, Cuba e Israel, a pesar de ser su principal aliado en el Medio Oriente" (Juventud Rebelde, 2013). Según los documentos de Snowden, los idiomas más requeridos por el interés de la seguridad son el ruso, español, árabe, chino, farsi, francés, alemán, coreano y portugués.

Como consecuencia de todas estas revelaciones, los actores involucrados en este caso pidieron explicaciones al presidente estadounidense por las interceptaciones ilegales y el espionaje atrevido de los organismos de inteligencia. El presidente, por el contrario declara que no tenía conocimiento de los hechos y por lo tanto no se disculpó como lo habían pedido algunos de los afectados.

La respuesta llegó el 17 de enero de 2014, en el Discurso que el presidente Obama ofreció acerca del análisis de la estrategia de la inteligencia de señales. Comentó que siempre los Estados Unidos se apoyaba de la inteligencia con el fin de proteger el país y la libertad, que durante la Guerra Fría fue necesario que el presidente Truman creara la NSA para penetrar el bloque soviético y neutralizarlo.

Tras la caída de Unión Soviética aumentaron las amenazas de grupos terroristas y Armas de Destrucción Masiva; la globalización e internet multiplicaron las posibilidades de más violencia, pero el hecho que disparó las alarmas para fortalecer las agencias de seguridad fue el 11 de septiembre.
A través de la "mayor cantidad de información digital y de supercomputadoras potentes les ofrece a las agencias de inteligencia la posibilidad de escudriñar masivas cantidades de datos (...) o encontrar alguna pista que pueda impedir amenazas inminentes. Es una herramienta poderosa" (Obama, 2014).

El presidente Obama afirmó:"no haré hincapié en las acciones o las motivaciones del Sr. Snowden; declararé que la defensa de nuestro país depende, en parte, de la fidelidad de aquellos a quienes se les encomiendan los secretos de nuestro país." (Obama, 2014).

Obama también agregó: respecto a la obtencion de "datos de inteligencia de señales, las agencias de inteligencia (...) utilizarán estos datos para satisfacer los requisitos específicos de seguridad, a saber: contrainteligencia, antiterrorismo, la lucha contra la proliferación, ciberseguridad, la protección de nuestras tropas y nuestros aliados" (Obama, 2014).

Estas son algunas de las declaraciones del Presidente Barack Obama del discurso ofrecido el 17 de enero de 2014.

La información es un arma de doble filo, y será siempre un elemento de vital importancia para los actores más importantes del Sistema Internacional. Las relaciones entre Estados serán condicionadas a los intereses que se tengan de por medio, y el tipo de confrontaciones y acciones efectuadas por el poder de la información serán tema de prioridad en las agendas nacionales, mientras la tecnología esté a la vanguardia y sea una herramienta imprescindible de los Estados.

\section{Conclusiones}

La evolución del espionaje y la tecnología desde la Guerra Fría se ha dado a través de las agencias de seguridad. Desde el 11-S se dio un giro a nivel mundial de cómo enfrentar el terrorismo y ya nada volverá a ser igual. Los métodos de guerra han cambiado sustancialmente por el crecimiento acelerado de las tecnologías en esta era digital que permite que nada quede fuera de su área de influencia.

Las Relaciones Internacionales se caracterizan por alianzas de acuerdo a intereses. A su vez cada Estado continúa desarrollando programas de vigilancia de alta tecnología como arma contra el terrorismo y como arma de control. 
Quién posee tecnologías de información, tiene en sus manos un arma muy poderosa, porque conoce a su enemigo y puede planear estrategias para neutralizar cualquier acción; pero Internet ya no es una herramienta segura para ningún gobierno y mucho menos para los ciudadanos, porque, el interés común es más importante que el interés particular, por lo que se cambió la privacidad por la Seguridad Nacional.

La Política Exterior es un área de inmensa competencia entre actores, haciendo que las naciones se sirvan de medios para controlar el crecimiento de otras naciones que no quieran servir a sus intereses y quieran desarrollarse rápidamente. Aunque es una vieja estrategia, el espionaje humano sigue siendo un medio utilizado para recopilar y analizar información. A su vez, el poder militar y económico ya no son suficientes, ahora, el Estado que tiene poder tecnológico, encontró una nueva arma poderosa a través del dominio de la información.

Ahora todos somos observados, la libertad fue reemplazada por la dependencia de la tecnología y los métodos de vigilancia en aras de la seguridad. Las nuevas tecnologías son controladas a través de los propios equipos que utilizamos día a día para comunicarnos y será muy difícil, si no imposible, intentar abandonar el uso de la tecnología que hoy nos rodea.

En las guerras de hoy, ya no se conoce al enemigo, ni qué estrategia se va a plantear, pues el ciberespionaje es un arma poderosamente efectiva, silenciosa, barata, no pone a nadie en evidencia, no es necesario el desplazamiento de ejércitos y se mueve a gran velocidad.

Por el auge del uso de la tecnología en la actualidad, nos hemos convertido en espías con nuestros equipos, a todos nos afecta la vigilancia en la vida cotidiana, por lo que hemos cambiado la privacidad por la seguridad, lo cual legitima el uso de la vigilancia.

\section{Referencias}

Barrientos Ramírez, F. (2010). El fracaso de la Comunidad de Inteligencia de Estados Unidos el 11 de septiembre de 2001: ¿Fallas humanas o sistémicas? Revista Política y Estrategia(116), pp. 43-85

Burghardt, T. (13 de julio, 2013). [En línea] Disponible en http:// www.globalresearch.ca/echelon-today-the-evolution-of-annsa-black program/5342646

Bush, G. W. (20 de septiembre, 2001). The White House. [En línea] Disponible en http://georgewbush-whitehouse.archives. gov/news/releases/2001/09/20010920-8. es.html de la Cámara, M. (22 de octubre, 2010). La Política Exterior de Rusia. Madrid: Real Instituto Elcano.

Duque Quicios, J. (2006). La comunidad de inteligencia estadounidense. Inteligencia y Seguridad(6), pp. 15-38.

Greenwald, G., y McAskill, E. (07 de junio, 2013). The Guardian. [En línea] Disponible en http://www.theguardian.com/ world/2013/jun/06/us-tech-giants-nsa-data

Juventud Rebelde. (30 de agosto, 2013). Juventud Rebelde. [En línea] Disponible en http://www.juventudrebelde.cu/internacionales/2013-08-30/estados-unidos-potencia-espionaje sobre-rusia-/

Maldonado, C. E. (Julio, 2013). La red Echelon: el control de internet y de todas las comunicaciones. Le Monde diplomatique, pp. 30-31.

Morales Hernández, J. (Mayo de 2003). Los Servicios de Seguridad e Inteligencia en la Rusia de Putin. UNISCI Discussion Papers, 22.

Morelli, A. (24 de junio, 2013). Apuntes para una charla sobre la administración del conflicto internacional en el ciberespacio. Argentina.

Obama, B. (2014). Discurso del presidente Obama sobre el análisis de la estrategia de inteligencia de señales. Washington D.C.

Pachón Ovalle, G. (01 de junio, 2004). La red Echelon: Privacidad, libertad y criptografía. (P. d. Barcelona, Ed.)

Polo, H. (2009). La nueva politica exterior Norteamericana. El espejismo Obama. El viejo topo (262).

Ramonet, I. (Julio, 2013). ¡Todos fichados! Corporación Viva la Ciudadanía.

Sánchez Medero, G. (2009). Internet: Una herramienta para las guerras en el siglo XXI. Revista Política y Estrategia, pp. 21-31.

Serra, F. (2008). Rusia ante, contra o con occidente:diferentes posiciones en las relaciones entre dos ejes complementarios en el poder mundial. UNISCI Discussion Papers(17), pp. 87-88.

Tovar Ruiz, J. (2009). La política europea de Barack Obama: tres meses de nueva relación transatlántica, p. 5.

Para citar este artículo:

Alba, D. (2014). El espionaje y agencias de seguridad: los Estados Unidos y la Federación Rusa. Ciencia y Poder Aéreo, Vol. 9 (1). Pp. 97-105 